\title{
Measuring Latency in Virtual Environments
}

\author{
Sebastian Friston and Anthony Steed
}

\begin{abstract}
Latency of interactive computer systems is a product of the processing, transport and synchronisation delays inherent to the components that create them. In a virtual environment (VE) system, latency is known to be detrimental to a user's sense of immersion, physical performance and comfort level. Accurately measuring the latency of a VE system for study or optimisation, is not straightforward. A number of authors have developed techniques for characterising latency, which have become progressively more accessible and easier to use. In this paper, we characterise these techniques. We describe a simple mechanical simulator designed to simulate a VE with various amounts of latency that can be finely controlled (to within $3 \mathrm{~ms}$ ). We develop a new latency measurement technique called Automated Frame Counting to assist in assessing latency using high speed video (to within $1 \mathrm{~ms}$ ). We use the mechanical simulator to measure the accuracy of Steed's and Di Luca's measurement techniques, proposing improvements where they may be made. We use the methods to measure latency of a number of interactive systems that may be of interest to the VE engineer, with a significant level of confidence. All techniques were found to be highly capable however Steed's Method is both accurate and easy to use without requiring specialised hardware.
\end{abstract}

Index Terms—Latency, measurement

\section{INTRODUCTION}

In the development of virtual environments (VEs), an important characteristic is latency. Defined as "the time lag between a user's action... and the system's response to this action" [21], latency has repeatedly been shown to reduce the effectiveness of virtual environments and other interactive computer systems on many levels. Latency can result in a discord between the proprioceptive cues of the user and the simulated stimuli. This conflict between experienced sensations and expectations learned over a lifetime results in reduced presence (the acceptance of the virtual stimuli as real) [25]. Its impact in this respect has been shown to be more significant than the difference between photo-real and non-photorealistic rendering $[31,15]$, while other studies have found it detrimental to performance in physical interaction [14], detrimental to performance in collaborative tasks [7], and that it is responsible for increased simulator sickness [4].

Those who have studied the effects of latency recommend VEs have no more than $17 \mathrm{~ms}$ [2] of effective delay, though others have found the effects of latency at even lower levels [11]. Constructing a VE with response times anywhere near these is difficult. Latency is a result of the accumulation of processing and transport delays inherent to the computer systems that constitute the VE. Further, measuring it is less than straightforward. It is this last point, that we aim to address.

A number of authors have presented techniques for measuring latency, and they have become progressively easier to use with less reliance on specialised hardware. Two of these techniques are Steed's Method, and Di Luca's Method. Their simplicity, low cost and low coupling to the configuration of the VE under test make them applicable to a wide range of interactive systems. The authors of those methods have taken care to confirm their results against alternative systems where available, but no objective characterisation of their accuracy exists. The aim of this paper is to provide the VE engineer with a reference they can use to select the most suitable measurement technique for their situation, with awareness of the confidence they may have in the results.

In our study, the two techniques above are used to measure latency in a controlled environment. In developing the test procedure, we create a mechanical simulator capable of simulating the visual stimuli our

- Sebastian Friston is with University College London.

E-mail: S.Friston@cs.ucl.ac.uk

- Anthony Steed is with University College London.

E-mail: A.Steed@cs.ucl.ac.uk

Manuscript received 12 September 2013; accepted 10 January 2014; posted online 29 March 2014; mailed on 1 May 2014. chosen methods will expect, with finely controlled latency. In addition, we develop an algorithm to perform frame counting automatically from high speed video, which is presented here as a third alternative.

We begin with a review of latency measurement techniques, before introducing our chosen techniques in detail, describing their operation and what information about the accuracy can be inferred from the original publications. We describe the Automated Frame Counting method, the motivation for machine-vision based automation of frame counting, and present our automated counting solution. A mechanical simulator, capable of simulating latency to within $3 \mathrm{~ms}$ is documented and its operation verified. Each measurement technique is used to estimate latency from motion actuated by this simulator. We discuss the results, investigating further where necessary to build an understanding of the performance of the techniques under different conditions, and what factors influence their accuracy. Finally, we use our results to characterise accuracies of the measurement techniques at a given confidence interval, and use them to measure the latency of various real interactive systems.

\section{Previous Work}

A number of latency measurement techniques have been developed. Some observe the VE in action and attempt to monitor latency in the same way it would be detected by the user. Others take advantage of, or even modify, the VEs design in order to characterise it. Latency measurement has been conducted on VEs from immersive acoustic environments to those employing haptic feedback devices, but most methods focus on assessing visual feedback $[17,9]$. We continue this trend as visual is the predominant stimuli in studies on the effects of latency. In this section we review the various ways latency has been measured in different interactive systems.

One way to characterise latency is to probe the connections between the components (hardware or software) that combine to produce the stimuli, assessing the delay of each stage individually. For example, in their investigation into the delay of cloud gaming systems Chen et al. hooked the EndScene() API of DirectX to ascertain the exact time spent by the application drawing the scene [5].

Miller et al., in their investigation of an immersive acoustic environment, placed calls to toggle a pin on the parallel port at certain points in the simulator software. These pins were monitored with an oscilloscope, along with output from the headphones and tracker hardware [16]. This was also done by Mine, who externalised receipt of the tracking data when assessing the total end-to-end delay of a VE, through an A/D converter [18]. This method provides good resolution. Miller, et al, were able to identify the impact on delay of various output buffer configurations, for example. However, it requires that the simulation be modified at a low level, and the accuracy is dependent on the actuation 
of the monitored events. In [1], Adelstein et al. measured how the latency of trackers varied with different accelerations. The trackers were attached to a mechanical arm fitted with a rotary encoder, which provided the ground truth. A software library read the PC's very precise hardware timer directly, in order to determine the delays of each stage in the communication paths between the encoder and tracker. In [16], Miller et al. performed a loopback test to confirm the response times of the parallel port used, but not all VE hardware may have this option.

The advantage of modifying the virtual environment system, is that the scene is independent making it possible to gather comparable performance data for varied virtual worlds. With VEs built from offthe-shelf modules, and modern operating systems further abstracting the hardware, this is becoming more difficult. Perhaps as a result most examples of measurement techniques are of the outside observer type. Here, a latency measurement system passively monitors the real and virtual world simultaneously and determines the latency from the discrepancies between the observations. One advantage of outside observer methods is that they assess the total time for the sensor data to be acquired or output data to be actuated, which probing may miss Another is that they are independent of the VE implementation. An example of this approach is by $\mathrm{He}$ et al., who monitored both a tracker and an object within a VE, with a single camera. The latency was derived by counting the frames between the movement of a real object and corresponding movement of the virtual object (the manual frame counting technique) [8].

Roberts et al., used the manual frame counting technique to measure the end-to-end delay of an immersive collaborative environment using synchronised cameras [22]. The frame counting technique is popular because cameras are cheap and readily available. However it has a number of drawbacks. The main drawback is that the resolution of the measurement will be limited to no less than the length of 1 frame. If the exposure time of the camera is large enough with respect to the frame rate of the output device, multiple output frames may be captured resulting in ambiguity in when exactly the output event occurred. All camera-based systems rely on the latency being higher than the Nyquist rate of the camera, or no latency will be detected at all One way to negate these issues is to simply use a high speed camera, though then the cost advantages diminish and due to the short exposure time, illumination becomes a concern [30]. Alternatively, there are techniques that derive latency independently of the frame.

Sielhorst et al., measured the latency of a video see-through AR system by encoding times in moving circles on a display. A camera recorded the display of the AR which contained both the true time and time as visualised by the latent AR system. The latency could then be 'read' by decoding the timestamps from the positions of the circles. This resulted in a resolution of $1 \mathrm{~ms}$ [24]. Steed developed the Sine-Fitting Method, where a camera monitored a (tracked) swinging pendulum and a corresponding virtual pendulum. Image processing algorithms fit the discrete locations of the targets from each frame on to two sine waves; the phase difference between them can then be used to calculate the latency. Steed obtained superior results to those achieved with a $500 \mathrm{~Hz}$ camera using the manual frame counting method [26]. Swindells et al., superimposed a virtual turntable on top of a real one in a virtual hand lab, and the latency was calculated from the angular difference between the two [27]. These techniques allow for sub-frame length resolutions. While they are all subject to motion blur or 'bleeding' of the output frames, this has little effect on the results due to the choice of image processing (Steed), encoding method (Sielhorst, et al) or capture hardware (Swindells et al.). The methods are still subject to the Nyquist rate however, and require constraints on the type of movement, and composition of the VE.

Alternative techniques employ other capturing devices. Mine's method utilises photodiodes to detect the passing of a motion tracker (attached to a pendulum) through a point, and a corresponding virtual object through an equivalent location on the display [18]. This requires the location of the photodiode on the display to be carefully calibrated however. Di Luca presents a method where two photodiodes, one attached to a tracker, and one to the screen, move across two greyscale gradients (one fixed relative to the moving tracker, the other virtual moving with the aforementioned tracker fixed relative to the diode). The signals output from the diodes are captured by the soundcard of a computer, normalised, filtered and processed with a Fast Fourier Transform (FFT) to find the maximal frequencies (those corresponding to the motion of the tracker); then as in Steed's Sine-Fitting Method, the phase shift determines the latency. The advantages of this method are no calibration requirements (as only the change in gradient expressed by each signal is considered) and the high sampling rate (potentially $44 \mathrm{KHz}$ ) will be considerably higher than current displays [6].

All the methods so far have required some modification to the simulation software or hardware, or addition to the virtual scene. For example Steed requires a scene containing a virtual pendulum while Di Luca requires a gradient. A third type requires no modification to the $\mathrm{VE}$ at all. An example of an entirely passive measurement is presented by Miller and Bishop. Their technique, the latency meter, utilises 1D CCD cameras (which can operate at much higher frequencies than regular 2D cameras) to identify the 'centre of brightness' of the scene they are observing. Two synchronised cameras, one facing the (tracked) user, the other the display of the VE, track the movement of this centroid and calculate the latency from the difference between the two. Much information is lost in this conversion of the scene to a $1 \mathrm{D}$ offset, and so a linear relationship between the two centroids cannot be relied upon. Instead Miller and Bishop use the velocity of the centroid (as when the tracker is not moving, the VE should not change significantly), matching 'stop events' between two signals and deriving latency from the time between them. This technique, while not requiring explicit modification of the VE, does rely on scenes where motion will result in large changes in light levels. The technique was verified against Mine's method [16]. There is a limitation however that the technique shares with all the aforementioned methods: it requires a sample of a number of movements.

$\mathrm{Wu}$ et al., attempted continuous latency measurement with two techniques: automated frame counting with a $1 \mathrm{KHz}$ camera, and an angular difference method with a low frequency camera. For the frame counting method, they implemented an algorithm that could identify two frames temporally separated but in which the position of a slider corresponded to the same location in both, this allowed continuous measurement to $1 \mathrm{~ms}$ resolution. For the angular difference method, they implemented an algorithm which assessed the angle of two bars and calculated the difference between them. Their measurements were accurate enough to reconstruct the apparent oscillation of the latency over time, due to the interplay between the different clocks of the actuator and the sensor [30].

\section{The Sine-Fitting Method}

The Sine-Fitting Method was introduced by Steed in 2008 in [26]. It is an outside observer type technique that facilitates latency measurement using commodity video cameras. With this technique, a salient object within the VE is configured to follow a tracker. Both the tracker and the object are captured in the same frame of low speed video, and machine vision techniques used to recover their motion. By ensuring that the tracker moves in a sinusoidal pattern, for example by attaching it to a pendulum, sine waves can be fitted to the motions sampled at low rates. The latency is then determined from the phase-difference between them, at values much lower than the time between frames. Steed used the Sine-Fitting Method to measure latency of different image generators for a CAVE and an Intersense IS-900 tracker. The sinusoidal motion was produced by attaching a tracker to a pendulum. Steed suggested the method may be improved by using cross-correlation (personal communication), which we investigate, so the method - measuring latency from the phase difference of two cyclical motions sub-sampled with low speed video - will herein be referred to as Steed's method, with two variants: sine-fitting and cross-correlation.

\subsection{Implementation}

In the reference implementation (based on code available from Steed ${ }^{1}$ ), two targets of different colours are used.

\footnotetext{
${ }^{1}$ Available at http://www.cs.ucl.ac.uk/staff/ucacajs/LatencyDemo/
} 
The video is cropped around each target resulting in two sequences. For each, the median is calculated over the entire sequence and then subtracted to remove the background. The sequence is then filtered on the colour of the target to reduce the influence of noise. The horizontal displacement of the target is then found by calculating the luminance centroid for each frame.

Once the motion is extracted for each target, it is smoothed and normalised to provide a set of samples characterising a sinusoid. A sine wave is fitted to each set of samples with least-squares non-linear curve fitting. This involves iterating to find the coefficients for a data-set, that minimise the difference squared, with respect to a reference set. In the Sine-Fitting Method, these coefficients are the frequency and phase which define a sine-wave (the data set), and the reference set it is compared with is the target motion.

The phase difference will be in fractions of a frame, and so knowing the framerate, the latency can be calculated to sub-frame length accuracy.

\subsection{Results}

Steed compared the latency estimations of the method with the estimations obtained using manual frame counting and found them far superior in resolution. The method was able to identify latencies of $11 \mathrm{~ms}$ (between two eyes in frame sequential stereo video). An attempt was also made to measure a difference of $3 \mathrm{~ms}$, being the difference between different points of the scan out of CRT projectors. No statistically significant conclusion could be drawn from this, with the author noting it could be due to limitations of the measurement technique or inherent variations in the latency of the system.

\section{Di LucA's Method}

Di Luca developed the method presented in [6] in order to characterise the effect of input frequency on VE latency. They note many techniques, including Steed's Method, use pendulums, which oscillate at only one frequency. The technique ${ }^{2}$ involves using two light-sensing devices to capture the positions of the tracker, and an object in the VE, synchronously. The tracker is attached to one device which moves across a gradient, the virtual object is another gradient, which moves in front of a static sensing device affixed to the display. The sensed luminance is proportional to the horizontal displacement across the gradient (position). The light-sensors are two photo-diodes, connected to the input of a PC sound-card. The cost of the equipment is low, and the sampling rates high. The tracker is moved in a roughly sinusoidal pattern by hand, and the luminance/displacement measured by each sensor captured. The waveforms are cross-correlated to determine the delay.

\subsection{Implementation}

The light-sensing equipment consists of two photo-diodes, and amplifier circuits which are arranged such that a light-dependent voltage source is provided by each. This is then connected to the input of an $\mathrm{A} / \mathrm{D}$ converter such as a sound-card. One concern with the use of a soundcard is that it is likely to have a high-pass filter. As Di Luca notes this is not usually an issue as the amplitude will be modulated by the refresh rate of the display (nominally $60 \mathrm{~Hz}$ ). In practice however we found that not all displays drive pixels black between frames. Displays with LED backlights such as those in laptops for example, maintain a constant luminance for unchanging visuals. In these cases it is necessary to reduce the brightness of the display and rely on the Pulse Width Modulation (PWM) of the backlight to oscillate the illumination of the entire screen.

Once suitable displays have been appropriated, standard audio recording software can be used to capture the change in luminance as the gradients move relative to their sensing devices. The samples are then normalised and filtered in a number of steps to recover the dominant waveform in each. The signals are first low-passed $(10 \mathrm{~Hz})$ to remove the modulating frequency that allows the signal to pass through

\footnotetext{
${ }^{2}$ The source code for the implementation is available at http://people.kyb.tuebingen.mpg.de/max/
}

the soundcard's high-pass filter. They are then windowed to remove the start and end periods. A Fast Fourier Transform is applied and from this the dominant frequency (assumed to be the user's movement) is retrieved. The signals are then band-passed by convolving them with a waveform of the dominant frequency. Now that the motions of the tracker and virtual object have been recovered, cross-correlation is used to identify the phase-shift between them.

\subsection{Results}

Di Luca did not compare the method's results to those obtained with another, but did modify the tracker configuration of the system under test to introduce predictable amounts of latency and confirmed the estimations responded appropriately. In one set of measurements a standard deviation of $5.1 \mathrm{~ms}$ was found, though again this is may be inherent to the VE.

\section{Automated frame Counting}

To compare the latency measurement techniques, we chose to characterise them by the error in their estimations. To determine error the ground truth must be known. In our experiments the ground truth is provided by a mechanical simulator, though in order to provide this, its operation must be verified. To do this we elected to perform frame counting. Manual frame counting as presented previously however is not practical for the number of captures our investigation will involve, or the level of confidence we require, so we present here a new algorithm to perform automated frame counting.

Frame counting involves capturing the system in operation, and identifying the samples at which two tracked objects display common and distinctive patterns of motion (such as starting and stopping, or reaching a specific point in space). The number of samples between them define the latency, in discrete units of time equivalent to the time between samples. This method can be applied to almost any system and, given an ideal capture, has a number of advantages including high robustness and invariance, and the ability to measure changes in latency during the capture.

In practice though, identifying where in the capture each event occurred is not straightforward. Given the small exposure times required for such high capture rates, illumination becomes a concern [30, 26]. Lighting fluctuations or slight instabilities in the camera mount or the tracked objects are manifested as small oscillations (noise) in the apparent position of the object in the video. As the frame rate increases the distance an object can move between two consecutive frames decreases until the difference between movement due to motion, and noise, becomes imperceptible. At $1000 \mathrm{fps}$ if an object is moving fast enough the SNR will be high enough to determine that the object is in motion, but this highly limits the range of motions that can be detected, and accelerations cannot be detected at all.

$\mathrm{Wu}$ et al., account for this by tracking the absolute normalised horizontal positions in space (instead of motion) and measure the latency by identifying the frames at which the targets reach a specific offset from the edge of the frame. This requires a perfect, known linear relationship between the tracker and target position however. Wu et al., managed this by using a video feed of the tracker as the actuator [30]. He et al., measure a real system using a similar technique but with a grid for guidance. Even with this though, and video captured at 60fps, they report it took 10 hours to manually perform the frame counting and suggest this process be automated with computer vision techniques [8].

\subsection{Implementation of an automated frame counter}

Our automated frame counting algorithm mimics the process of manual frame counting: identify a distinctive pattern of motion, and then identify at which frame this began. Similar to Steed, and Wu et al., we use image processing techniques to extract the horizontal positions of the tracked objects throughout the capture, resulting in a set of samples (a signal) that characterises the motion of the object. Identifying a distinctive pattern then becomes a problem of feature detection in the single dimension. We nominate a motion feature with high distinctiveness and localisation, and utilise feature detection algorithms to perform the frame counting. 


\subsubsection{Capture setup}

The setup for the Automated Frame Counting method is similar to that of Steed, and Wu et al. A high speed camera is positioned to capture both the tracker, and a virtual object, in the frame. The virtual object should move in free space with the tracker. The algorithm extracts foreground objects based on luminance, so the virtual object and any marker attached to the tracker should be bright and distinctive compared to the background. Once a capture of the targets in motion has been taken, the algorithm will track their locations throughout the video identifying distinctive motions, and when they occurred, for each target.

\subsubsection{Extracting motion from video}

The signal we perform feature detection on is the set of horizontal positions of a target over the entire capture. To extract these, the video is filtered such that the background disappears and the targets remain as solid clusters of pixels - binary blobs. The centroids of these are then tracked throughout the sequence. First, each frame of the video is grayscaled to get a single luminance value for each pixel. The histogram of the first frame is then bi-segmented, clustering the luminance for each pixel into one of two classes. All frames are binarised based on one threshold. The user has the option to define the threshold. If they choose not to, one is automatically calculated using Ostu's method Ostu's method selects a threshold such that the inter-class variance of the luminances is minimised [20]. This method was selected as it is simple and has been shown to be consistently among the better performing thresholding methods [23].

Once all frames have been processed the user is presented with the first frame again in order to nominate the positions of the objects to track. For each frame in the sequence, all the binary blobs are identified. For each object, the closest blob to the last known position is considered to be the object in the current frame. The centroids are extracted and the last known positions updated, and the process repeats on the next frame This heuristic is simple but due to the rate of capture is robust and operates without the need for filtering or prediction. This method relies on the assumption that the captures are taken of monochromatic targets in environments with very little background noise. This is reasonable because the algorithm was developed to assess latency measurement techniques and throughout these experiments the environment can be completely controlled. Should this be used to measure other VEs built with a wide range of different hardware, the assumption is unlikely to hold. A possible extension would be to replace the tracking of binary blobs with that of image patches, which would allow this method to measure VEs without any need to modify the scene, as any visually distinctive object within it would suffice.

\subsubsection{Extracting features from motion}

Our algorithm operates by identifying a specific feature in motion. That is, a pattern of displacements over time. We define the feature as the peak of acceleration. This is the point at which the velocity is zero, as the object changes direction in side-to-side motion. It was chosen as it is highly salient, distinctive (the acceleration before and after is likely to be high) and highly localised (the object will be still for smallest amount of time permitted by the laws of physics) meeting much of the criteria of the ideal local feature [28]. It also corresponds to the point in a user's motion that they are most likely to detect a discrepancy in the simulated stimuli. Users are more sensitive to differences in velocity than acceleration, so latency is most visible at the end of a movement when the latent VE continues to a display fast moving stimuli relative to the user's quickly decelerating head or limb [10]. With this feature the problem becomes that of edge detection in one dimension.

The detector must consider motion features (edges) with a large size relative to that of an individual sample. The user's speed will determine how many samples their motion is completed within (the feature size), and therefore a scale-invariant feature detector is required. We use an edge detector introduced by Lindeberg which uses a first-order Gaussian differentiating kernel applied at different scales, along with an edge strength measure, to allow for automatic scale selection [12]. Our kernel is given by Equation 1, where $s$ controls the width of the function relative to the kernel scale and is defined as $s=$ kernelscale $0.25 \cdot$ widthscaling factor.

$$
g(x ; s)=\frac{-x}{s^{2}} e^{\frac{-x^{2}}{2 s^{2}}}
$$

The signal we convolve this kernel with is the set of positions of our target over time. The result is the derivative of the signal, but calculated for the total displacement over a set of samples, averaged with a Gaussian function. The number of samples depends on the size of the kernel. A Gaussian kernel was chosen as it was found by Canny to be optimal in edge detection [19].

Averaging destroys local features which can be used to identify the exact sample containing the peak of motion. To mitigate this, we perform the convolution with functions of two different sizes as demonstrated by Bao et al., based on the observation that as the scale increases, noise is attenuated far more rapidly than the signal [3]. In our implementation, the kernel size (scale) remains constant and the width of the function is altered (with scaling factors of $1 \& 0.5$ ). The product of the convolutions is a filtered signal which maintains high locality while still permitting the algorithm to use averaging in order to make the gradient a useful property for feature detection.

To extract features from this convoluted signal we use non-maximum suppression as defined by Canny, selecting the sample at which the gradient assumes local maxima [29]. We define our own feature strength measure which is based on the area under the potential feature. We approximate the integral of this potential feature by summing the normalised magnitudes of the gradient at each sample within a window centred on the feature. If this sum is within a range, the feature is considered salient. The range is based on the shape of an ideal feature. Its upper value is half the window, biased slightly to allow for nonideal waveforms. The window size and kernel scale are interdependent, where the kernel scale is $4 \cdot$ windowsize +1 .

To prevent the use of different scales on different targets introducing error, a single scale which best represents the scale of the features in both is chosen. This is done by performing the convolution for a set of scales on each signal, and identifying the number of detected features at each scale. The scales were selected so the window sizes ( 10-1000 samples) spanned the periods of all the expected movement frequencies $(1-4 \mathrm{~Hz})$. The subsets for which the number of detected edges is consistent between the two signals is extracted, and from these, the set with the largest numbers of consecutive scales is identified. As aliasing may cause the signal to resonate at multiple scales, the scale set selection is guided by estimating the frequency (number of features) based on zero crossings of the normalised signal.

The scale used to detect the edges is selected from near the end of the set. Intuitively, the less averaging performed the better the localisation, however we discovered in simulation that the error was minimised when the scale was maximised, and the ideal scale is that which approximates the size of the average feature. In the final stage, the convolution is performed at this chosen scale on both signals, the feature points extracted as described, and the latencies calculated by subtracting the two feature point positions from each other.

This method of determining latency from the difference between two discrete positions in time is similar to that of $\mathrm{Wu}$ et al., but where they identify when each target reaches a specific displacement in space, we identify when each achieves a maximal acceleration. This makes fewer requirements of the VE as a linear spatial relationship between the two targets is not needed.

\subsection{Measuring latency with the automated frame counter}

An implementation of the Automated Frame Counting method was created. The user begins by selecting a viewpoint in the virtual world, and positioning the tracker and camera as described in section 5.1.1. Figure 1 shows an example of a suitable setup. In our experiments the tracker was moved in a sinusoidal pattern for $\sim 3$ seconds by hand.

The user provides our software with the video file. The software guides them through selecting the threshold for binarising the frames, and identifying the locations of salient objects to track. Once tracking is complete the user selects the feature scale. To do this they are presented 


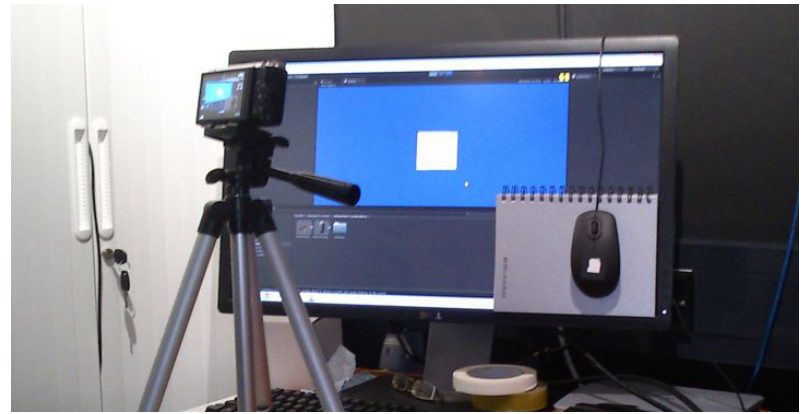

Fig. 1. Example setup for the Automated Frame Counting method. The mouse is the tracker and the VE is a Unity set.

with a plot of target positions. The features detected at the current scale are indicated on the plot. The user increases or decreases the size of the scale until the number and location of the detected edges matches those suggested by the plot. The algorithm then extracts the features at this scale. Subtracting the feature locations of one target from the other provides the number of frames, and thus the latency, between them. The average of these is returned as the latency estimate for that capture.

\subsection{Verifying the algorithm}

Pairs of sinusoidal waveforms were generated with sample rates equivalent to the frame rate of the high speed video, and Gaussian noise introduced, at levels designed to mimic that encountered when recovering motion from the video. One of the sinusoids of each pair was shifted to emulate latency. Waveforms of frequencies between $1-4 \mathrm{~Hz}$, and latencies between $0-120 \mathrm{~ms}$ were generated. The frame-counting algorithm was then used to estimate the latency of the pairs. (From here on in, simulated will refer to waveforms with controlled latency but that have been recovered using the sensing devices \& algorithms of the aforementioned techniques, while virtual refers to those that have been entirely procedurally generated to mimic them.)

\subsection{Results \& Failure Cases}

The Automated Frame Counting algorithm estimates latencies between corresponding motion features in the position samples of two targets extracted from each capture. For each capture, a number of latencies will be estimated, the number depending on the frequency of motion and length of sample. For example, the virtual 'captures' all had lengths of 3 seconds, so for motion at $1 \mathrm{~Hz}, 6$ estimations would be made. The mean of these estimations is the estimated latency for that capture. This figure is the one most comparable with the other techniques, all of which estimate the average latency across the capture.

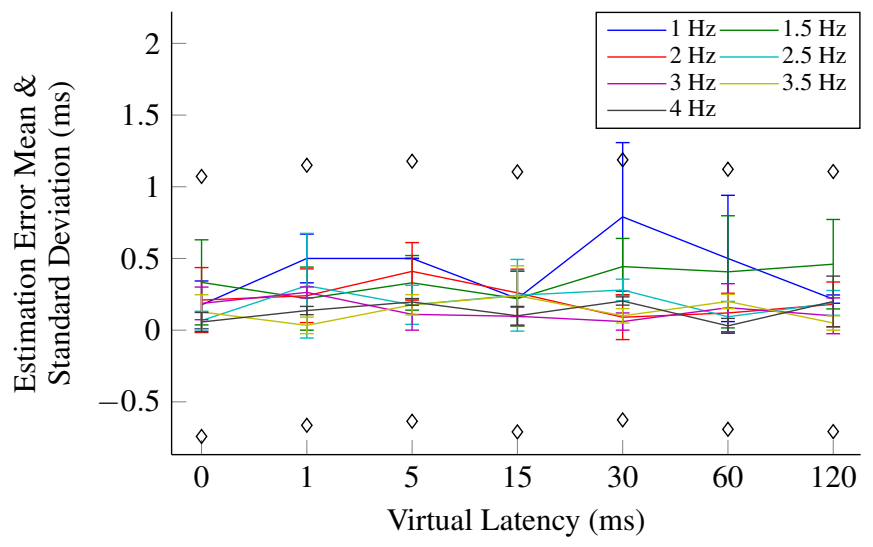

Fig. 2. The error in the estimates of latency in virtual waveforms made by the Automated Frame Counting method, for a range of latencies and frequencies
For each latency and frequency combination, three waveforms were generated, the latencies estimated, and the error of these estimations determined. The mean and standard deviation of these errors across the three estimations are shown in Figure 2. The standard deviation of the errors in the individual frame-to-frame latencies (the mean of which defines the latency estimation for that capture) are also shown in Figure 2, with diamond markers. For clarity, these data points show the average across estimations for all frequencies at the given latency.

The least robust part of the algorithm is the automatic scale selection. The reason for this is that it operates by first attempting to determine the number of motion features in the video and then selects a scale which resonates with this. This makes the scale selection more reliable, but also makes assumptions about the characteristics of the features it is trying to find that may not hold, especially for user generated motion. The algorithm includes a stage after automatic scale selection, permitting manual verification and correction, it is recommended that this is always used even if adjustment is rarely required. During verification this was needed even with the perfect sinusoids, as in some virtual waveforms the randomly generated noise was significant enough to confound the feature count estimation.

Another likely source of error is in the latency estimation stage. This occurs after feature location extraction, and during which the feature locations from one waveform are subtracted from the other. This simplistic method does not ensure the motion feature locations being subtracted correspond and it is therefore possible to subtract the wrong locations from each set. This may occur if a feature is detected in the latent signal, before the first feature in the primary signal, for example. This again can be avoided by verifying the detected features in the scale selection stage.

Finally, the object tracking algorithm has a limitation where if a tracked object is not visible in a frame, it will lose it for the remainder of the sequence and begin tracking another target - possibly the counterpart leading to erroneous latency estimations of zero. This can occur for example on displays which use Cold-Cathode Fluorescent Lamp (CCFL) back-lights or those with LEDs utilising PWM to control the brightness. This limitation needs to be corrected by using a more sophisticated algorithm that will take the entire sequence into account as it severely limits the applicability of this technique to real systems. For this investigation latency estimations were done on systems using CRT displays, or LED backlit displays with the brightness set to $100 \%$.

Once the correct scale has been identified however the algorithm is highly reliable. Out of over 2000 individual latency estimations performed on virtual waveforms, the largest deviation from ground truth was $4 \mathrm{~ms}$. The mean of a set of estimations will characterise the latency to under $1 \mathrm{~ms}$ with a CI (confidence interval) of $99 \%$ (calculated from the estimations of all latencies for all frequencies with a Critical (Z) Value of 2.58).

\subsubsection{Characterisation Metric}

To characterise and compare the measurement techniques, we chose to use the confidence interval of the estimation error at $99 \%$. Given a population, such as a set of estimation errors, this metric determines a range that any future member of the population will fall within, with a given probability, based on previous observations. By calculating the $\mathrm{CI}$ in terms of estimation error, we define the error margin that an end user can expect for their measurement with a high level of confidence. This is an intuitive characterisation of accuracy that can be used to compare the techniques. Throughout our experiments we determined estimation error for a range of frequencies and latencies so that any interaction between these variables and accuracy could be detected. The CI was always calculated in the same way, from the complete set of these, to ensure the characterisations are applicable under a wide range of conditions and directly comparable.

\section{Mechanical Latency Simulator}

To measure the accuracy of latency measurement techniques, we created a mechanical simulator which would mimic an interactive system as seen by the 'outside observer' camera, using targets actuated by mechanical devices, the latency of which could be finely controlled. Two 
Hitec HS-303 RC Servos were connected to an FPGA development board. RC Servos are driven by a $50 \mathrm{~Hz}$ PWM signal where the $\mathrm{T}_{\text {on }}$ period defines the angular offset of the servo's shaft from its neutral position. The servos we used supported a duty cycle of $1500-1900 \mu$ s for a range of $90^{\circ}$. Our system accepted position samples from a desktop computer and used them to define the duty cycle of the signal transmitted to the servos. This signal could be delayed for one servo, by a set amount of time, or as a function of time, by sending delay samples from the desktop. Delaying the waveform was achieved by sampling it at $256 \mathrm{KHz}$, converting it into a 1 bit data stream, which was fed into a FIFO buffer. The stream received by the servo was not the output of the buffer but a specific offset into it. The size of this offset determined the delay, and allowed the delay to be decreased instantaneously at any point during the simulation. $256 \mathrm{KHz}$ results in a period of $4 \mu \mathrm{s}$ which is well within the deadband of the servos used (that is, the resolution of the change in duty cycle that would cause the servos to move). By using an FPGA the control system for the servo could operate at far higher rates than one driven directly by a PC, permitting very high resolutions. Our control system is similar to that of Adelstein et al., designed to actuate trackers with varying frequencies and amplitudes using a mechanical arm. In their system a motor controller configured a function generator, which drove a DC motor with smoother sinusoids than the motor controller itself was capable of generating [1]. Servos operate by using a potentiometer as a rotary encoder and drive a DC motor connected to the shaft through a gearing system back or forth to match the intended rotation based on the impedance of the potentiometer. Therefore, servos are more vulnerable to manufacturing tolerances and wear than other options such as stepper motors. Out of four servos, only two had performance characteristics similar enough to actuate the targets with a temporal resolution of $1 \mathrm{~ms}$. If an equivalent mechanical simulator is built it may require the designer to try multiple servos, or perform calibration.

\subsection{Verification}

The mechanical simulator was used to actuate sinusoids between $1-4 \mathrm{~Hz}$ at latencies between $0-120 \mathrm{~ms}$. The latency was estimated with the Automated Frame Counting method. The motion for each combination was repeated three times.



Fig. 3. The actuation error of the mechanical simulator measured with the Automated Frame Counting method for a range of latencies and frequencies

Figure 3 shows the mean and standard deviation of the actuation error. The standard deviations for frame-to-frame latencies within each capture are again averaged for each frequency and illustrated with diamond markers.

The maximum error out of all estimations in all captures was $8 \mathrm{~ms}$. For a set of oscillations over a three second period, we can simulate latency on average to within $3 \mathrm{~ms}$ with a confidence interval of $99 \%$ (calculated from estimations of all latencies for all frequencies, with a Critical (Z) Value of 2.58 ( $\sim 2 \mathrm{~ms})$, including possible estimation error of the Automated Frame Counting method (1ms)).

\section{Results}

The mechanical simulator described above was used to simulate user motion and latent feedback, at frequencies of $1-4 \mathrm{~Hz}$ with delays of $0-120 \mathrm{~ms}$. In all simulations performed to assess the techniques, high speed video (1000fps) was taken to verify simulator operation. For each combination of frequency \& latency, the targets were oscillated for three seconds, three different times. The latency was estimated for each of these and the error (the deviation from the simulated latency) calculated. Any time the estimation error of a technique under test rose above $3 \mathrm{~ms}$ (the level of confidence we have in the mechanical simulator) the high speed video was reviewed. At no time did this show an error was due to incorrect simulator operation.

\subsection{Steed's Method}

To assess the accuracy of Steed's method, the mechanical simulator was fitted with two glossy targets. The motions were captured with a Panasonic HD camcorder at 25fps.

The Sine-Fitting Method was modified, replacing the region centroid based tracker with the binary blob based tracker used in the Automated Frame Counting method. Reviewing the horizontal motions recovered with each method had revealed the blob based tracker had less noise and jitter. Steed suggested the method may be improved by using crosscorrelation, in place of sine-fitting with least squares. Without requiring the movement to emulate a perfect sinusoid this is more tolerant of discontinuities in movement, frequency and of quantisation noise (in the time domain). The code provided by Steed was modified to perform latency estimation using both sine-fitting and cross-correlation so that they could be compared.

Video was captured at a resolution of $1920 \times 1080$. This was then down-sampled and the measurements (including tracking) repeated at 960, 480 and 240 lines to check the significance of the effects of (spatial) quantisation noise. Initial results suggested an interaction between spatial resolution and accuracy for both methods (Table 1), and movement frequency and accuracy for the sine-fitting variant (Table 2). Table 1 shows the mean, standard deviation, maximum and minimum error in all estimations for all frequencies and latencies performed at the displayed resolution. Table 2 shows the mean and standard deviation for all latency and resolution combinations, for each movement frequency.

Table 1. Estimation error of simulated latencies with Steed's method at various resolutions

\begin{tabular}{|c|c|c|c|c|}
\hline \multirow[t]{2}{*}{ Resolution (lines) } & \multicolumn{4}{|c|}{ Error (ms) } \\
\hline & Mean & Standard Deviation & Minimum & Maximum \\
\hline \multicolumn{5}{|c|}{ Sine-Fitting Method } \\
\hline 1920 & 11.67 & 10.19 & 0.18 & 85.09 \\
\hline 960 & 3.99 & 6.46 & 0.01 & 72.16 \\
\hline 480 & 6.09 & 8.83 & 0.09 & 10.00 \\
\hline 240 & 7.22 & 10.95 & 0.04 & 12.92 \\
\hline \multicolumn{5}{|c|}{ Cross-Correlation } \\
\hline 1920 & 7.76 & 3.96 & 0.00 & 22.00 \\
\hline 960 & 2.06 & 1.66 & 0.00 & 9.00 \\
\hline 480 & 2.21 & 1.49 & 0.00 & 8.00 \\
\hline 240 & 1.98 & 1.36 & 0.00 & 1.00 \\
\hline
\end{tabular}

\subsubsection{Steed's Method: Spatial Resolution \& Accuracy}

From Table 1 we can see the results are consistent for cross-correlation for all frequencies and resolutions, with the exception of 1920x1080 which shows a marked decrease in accuracy. That the other resolutions show no interaction with accuracy suggests that this is due to the object tracking rather than position resolution. The recovered positions for videoed targets at 1920 and 960 were taken and quantisation noise introduced by dividing the displacement samples and rounding down. The latency was estimated for a number of divisors.

Figure 4 illustrates the influence of spatial quantisation noise. The plots show the sum of the mean error and one standard deviation so neither metric can provide a false sense of confidence. The error and 
Table 2. Estimation error of simulated latencies with Steed's method at various frequencies

\begin{tabular}{|c|c|c|c|c|c|c|}
\hline \multicolumn{7}{|c|}{ Error (ms) } \\
\hline & \multicolumn{5}{|c|}{ Frequency } & \multirow[b]{2}{*}{ Varying } \\
\hline & 1 & 1.5 & 2 & 3 & 4 & \\
\hline \multicolumn{7}{|c|}{ Sine-Fitting } \\
\hline Mean & 3.86 & 4.84 & 3.13 & 7.94 & 9.05 & \\
\hline $\begin{array}{l}\text { Standard } \\
\text { Deviation }\end{array}$ & 4.60 & 7.40 & 3.66 & 18.05 & 11.83 & \\
\hline \multicolumn{7}{|c|}{ Cross-Correlation } \\
\hline Mean & 0.59 & 1.63 & 2.70 & 2.46 & 2.19 & 2.95 \\
\hline $\begin{array}{l}\text { Standard } \\
\text { Deviation }\end{array}$ & 0.50 & 1.30 & 1.29 & 1.49 & 1.81 & 2.62 \\
\hline
\end{tabular}

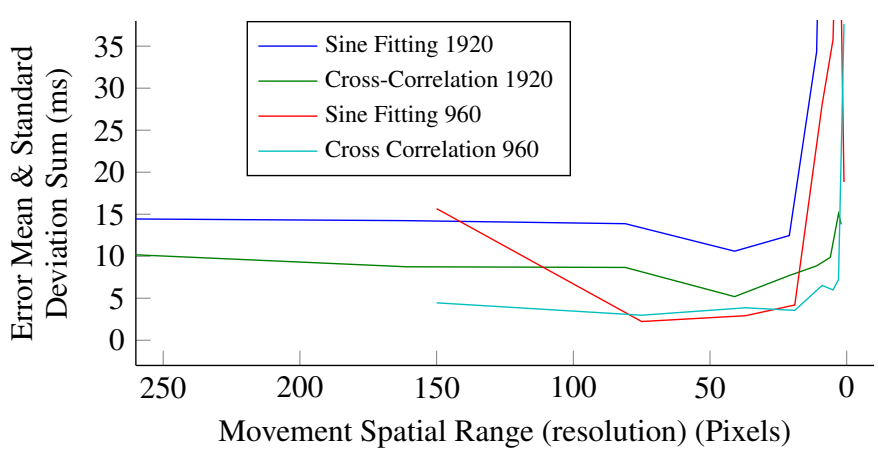

Fig. 4. The sum of the mean and one standard deviation of the estimation error for all latencies and frequencies, for estimations performed at various video resolutions by Steed's method

standard deviation, the sum of which is shown, are the mean of those calculated for all six latencies, when oscillating at $2 \mathrm{~Hz}$. The tracked positions from two captures were divided, the first captured at 1920 lines, the other at 960 . For each capture, the divisor was increased by the power of two each step until the range became so small latency estimation could not be completed.

Figure 4 demonstrates that spatial resolution has little impact on the accuracy of the estimations. A spatial resolution of as little as 20 pixels is sufficient to recover the latency to within 6ms (CI 99\%). Both sine-fitting with least-squares and cross-correlation have a minimum resolution below which they are unable to reliably recover the latency, cross-correlation has a slightly lower threshold.

From Tables $1 \& 2$ we can see that cross-correlation has consistently higher accuracy than sine-fitting with least-squares. Table 2 also suggests that the Sine-Fitting Method may be less reliable at higher frequencies, possibly due to temporal quantisation noise.

\subsubsection{Steed's Method: Temporal Resolution \& Accuracy}

To investigate how temporal resolution could affect the accuracy of the estimations, pairs of waveforms at frequencies between $1-4 \mathrm{~Hz}$ where generated with rates of 1000 samples/s, with the same latencies $(0-120 \mathrm{~ms})$ as used in previous tests. These were then subsampled to emulate captures at framerates between 5 and 1000fps.

Figures $5 \& 6$ show how the mean error of both estimation methods vary with framerate, for a set of seven frequencies (again, these are the errors over a number of latencies from 0 to $120 \mathrm{~ms}$ ). In both cases the the accuracy is invariant of the framerate until it is a low enough value such that the motion cannot be accurately expressed and estimation fails. This value is frequency dependent. Again cross-correlation demonstrates more consistent and higher accuracy.

By introducing spatial quantisation noise above, we have shown that not only is accuracy invariant with spatial resolutions above a certain threshold, but that this threshold is considerably small. Therefore high speed video can be used to verify the interaction of temporal resolution and accuracy. The high speed video was subsampled by extracting

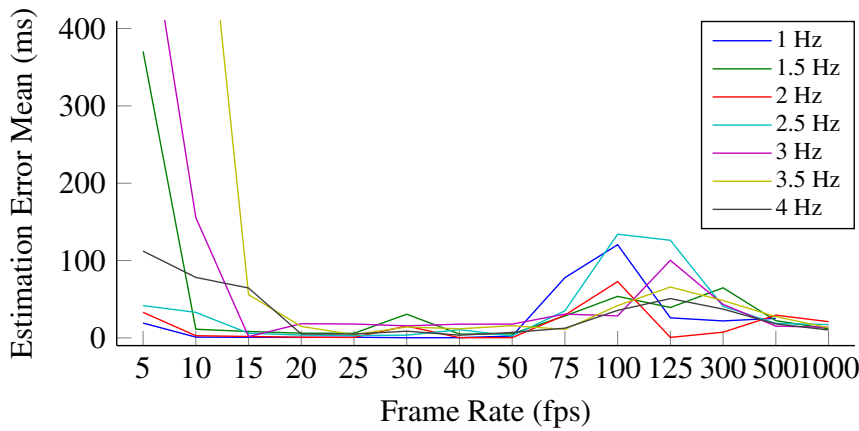

Fig. 5. Mean estimation error of the sine-fitting variant over all latencies, at all frequencies, for estimations performed on videos of various framerates

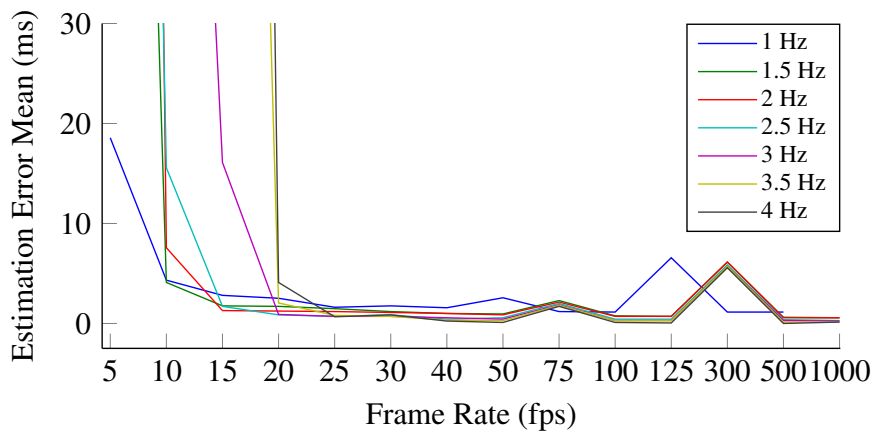

Fig. 6. Mean estimation error of the cross-correlation variant over all latencies, at all frequencies, for estimations performed on videos of various framerates

frames at set intervals, to provide the Sine-Fitting Method with higher rate captures at $3 \& 4 \mathrm{~Hz}$. Corroborating the results from our virtual data, the accuracy of cross-correlation remained unaffected, and the estimations from sine-fitting were not improved.

Since the noted error at higher frequencies is not a result of framerate, and does not occur in the estimations of virtual waveforms or in the estimations using cross-correlation, we conclude it is a result of the imperfections of the actuated waveforms, in which the sinusoid appears to skew slightly as the servos are driven towards their limits by the high rates of motion which confounds the sine-fitting process.

We also subject both methods to a waveform consisting of a set of sequential single cycles at increasing frequencies, to emulate how a user's manual motion may vary in speed. As expected the least-squared fitting function is unable to resolve to a single frequency.

Figure 7 shows the mean, and standard deviation of the errors, for the various combinations of frequency and mechanically simulated latency. The figures are averaged for all resolutions except 1920, the results of which were considered invalid. The plot does not display the estimations made by the Sine-Fitting variant of the $4 \mathrm{~Hz}$ or varying waveforms. The cross-correlation variant results are shown by the solid lines, while those of the sine-fitting variant are shown by dashed lines. Each frequency/latency combination was simulated three times, and the standard deviation shown is of the estimations for these three.

\subsubsection{Steed's Method: Conclusions}

We have demonstrated that Steed's method, both the sine-fitting and cross-correlation variants, can have accuracies as high as $\sim 12 \mathrm{~ms}$ and $\sim 7 \mathrm{~ms}$ respectively (best-case CI of $99 \%$ when the tracked motion is perfectly sinusoidal). When testing the interaction of resolution (spatial range) and accuracy we encountered anomalous results. Investigating this interaction further however revealed no relationship between this variable and accuracy of the estimation algorithm. Instead the algorithm was proved to be highly robust to quantisation noise in the time and 


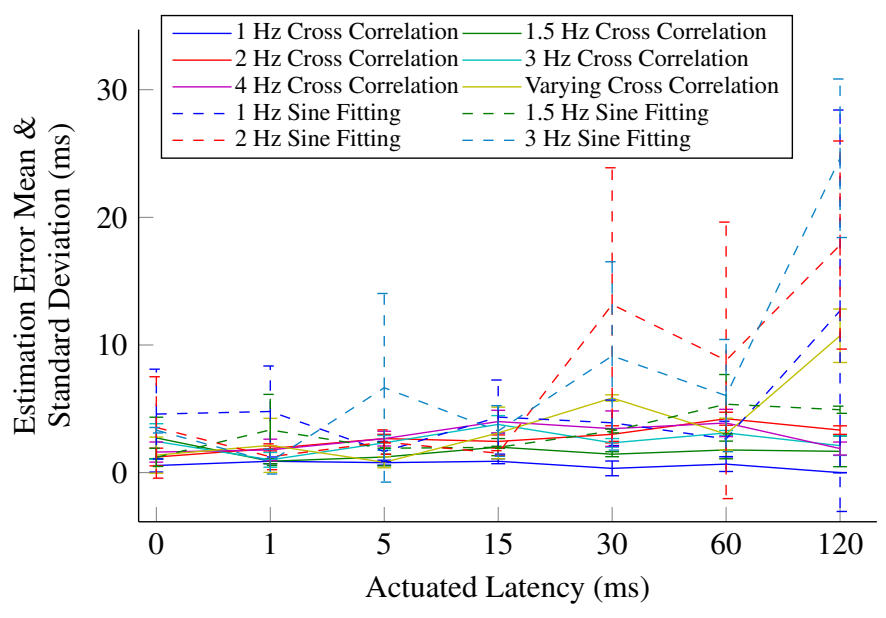

Fig. 7. The error in the estimations made by both variants of Steed's method, for multiple latencies and frequencies

spatial domains, and we determined guideline minimums for both. The anomalous results are likely due to errors introduced by the tracking code. The cross-correlation variant has proven to be significantly more reliable and consistent in its performance than sine-fitting using leastsquares so this variant will be used henceforth.

\subsection{Di Luca's Method}

To assess Di Luca's Method the capturing hardware was built as described and the diodes attached to the arms that actuated the targets in prior simulations. These were placed against an LCD display showing a single gradient. The arms were side by side as before so each diode viewed a distinct horizontal section of the gradient.

The arms were actuated at frequencies between $1-4 \mathrm{~Hz}$, with latencies between $0-120 \mathrm{~ms}$. Di Luca's Method includes stages to allow manual correction of the derived dominant frequency and correlation, however we found no corrections were needed. The errors in the estimations (mean and standard deviation) are displayed in Figure 8.



Fig. 8. The error in the estimations made by Di Luca's Method, for multiple latencies and frequencies

Computing the error mean $(2.8 \mathrm{~ms})$ and standard deviation $(1.9 \mathrm{~ms})$ for all latencies, for all frequencies allows us to determine an accuracy of $\sim 8 \mathrm{~ms}$ with a CI of $99 \%$ for this method.
Table 3. Estimation technique accuracy (ms) with a $99 \% \mathrm{Cl}$

\begin{tabular}{|c|c|c|c|c|}
\hline & \multicolumn{4}{|c|}{ Estimation Error Characteristic (ms) } \\
\hline \multirow[t]{2}{*}{ Characteristic } & & Method & & \\
\hline & $\begin{array}{l}\text { Mechanical } \\
\text { Simulator }\end{array}$ & $\begin{array}{l}\text { Automated } \\
\text { Frame Counting }\end{array}$ & Steed & Di Luca \\
\hline Mean & 0.64 & 0.23 & 2.08 & 2.79 \\
\hline Standard Deviation & 0.47 & 0.21 & 1.79 & 1.90 \\
\hline Minimum & 0.00 & 0.00 & 0.00 & 0.00 \\
\hline Maximum & 2.00 & 1.17 & 9.00 & 9.00 \\
\hline Accuracy at $99 \%$ CI & 1.86 & 0.78 & 6.69 & 7.68 \\
\hline
\end{tabular}

\subsection{Summary}

Table 3 attempts to illustrate the confidence one may have in any technique or the mechanical simulator. The figures were calculated from the set of error values for all latencies and all frequencies for each method. Error values are defined as the absolute deviation from ground truth (the simulated value). The error values for the mechanical simulator entry were measured with the Automated Frame Counting method. The values for the Automated Frame Counting method were calculated from estimations performed on virtual waveforms. For Steed's method the figures for all resolutions were included, except for the 1920 line captures which were considered invalid. The confidence interval was calculated with a Critical (Z) Value of 2.58.

\section{The MEASURED LATENCY OF REAL SYSTEMS}

After benchmarking the methods we attempted to determine how their estimations compared when measuring real interactive systems. We selected a set of systems chosen to represent both a range of interaction paradigms for which latency is important, and a range that will have high variance in performance.

The results are summarised in Table 4. For the Automated Frame Counting method, the maximum \& minimum latencies from the set of estimations for a given capture are included to illustrate the variations in latency of real systems. In addition to frame counting, cross-correlation was performed on the recovered positions from the high speed video. This further confirmed our findings into the invariance of accuracy with temporal resolution, as the differences between these results and those of Steed's method are marginal and within measurement error.

As evidenced by the range determined by Automated Frame Counting, real VEs, especially those running on PCs which are tasked with other concurrent responsibilities, are highly complex, and a great number of factors will determine performance. The measurements in Table 4 have been performed in sets designed to illustrate the performance impact that may be expected from changing one parameter of various configurations. It should not be considered as an accurate characterisation of the absolute performance of any of the included components or technologies. When considering the maximum \& minimum latencies for a given measurement it is also worth recalling that the automated frame counting estimation (for which the confidence interval in Table 3 is calculated) is the mean of the set of estimations. Larger errors may be encountered within that set (up to $4 \mathrm{~ms}$ was found during the verification stage).

In some configurations (*), a gradient could not be made available for Di Luca's Method, so results for these are not present. In another configuration (**), we used a Bluetooth tracker which was designed to control the cursor with pointing motions and therefore the mapping between lateral physical movement and motion on screen made it difficult to capture the tracker with a gradient. This is a limitation of our code, not Di Luca's Method, so although a result was derived it was considered invalid and omitted.

For the tests on Desktop 1, two demonstrations were built that displayed a white circle or gradient moving with the input device. One used a PictureBox control on a Winforms application, the other displayed a textured quad in an OpenGL (SharpDX) viewport. Both were written in C\# using .NET. For the Unity measurements, an environment containing a textured plane was created. The prefabs included in the 
Table 4. Latencies of interactive computer systems estimated with various methods

\begin{tabular}{|c|c|c|c|c|c|c|}
\hline \multirow[t]{3}{*}{ System } & \multicolumn{6}{|c|}{ Latency Estimate (ms) } \\
\hline & \multicolumn{4}{|c|}{ Automated Frame Counting Method } & \multirow[t]{2}{*}{ Steed's Method } & \multirow[t]{2}{*}{ Di Luca's Method } \\
\hline & Mean Latency & Maximum & Minimum & $\begin{array}{l}\text { Cross-Correlation } \\
\text { (1000fps) }\end{array}$ & & \\
\hline PC 1 Mouse Aero On & 30.00 & 45.00 & 11.00 & 29.00 & 32.00 & $*$ \\
\hline PC 1 OpenGL Windowed Aero On & 58.78 & 64.00 & 53.00 & 52.00 & 51.00 & 61.00 \\
\hline PC 1 OpenGL Windowed Aero Off & 24.00 & 32.00 & 18.00 & 22.00 & 22.00 & 23.00 \\
\hline PC 1 OpenGL Full Screen Aero On & 49.83 & 60.00 & 23.00 & 45.00 & 44.00 & 47.00 \\
\hline PC 1 OpenGL Full Screen Aero Off & 24.00 & 33.00 & 13.00 & 22.00 & 21.00 & 12.00 \\
\hline PC 1 Winforms Aero On & 58.47 & 66.00 & 50.00 & 53.00 & 51.00 & 40.00 \\
\hline PC 1 Winforms Aero Off & 34.64 & 44.00 & 29.00 & 31.00 & 32.00 & 22.00 \\
\hline PC 1 Winforms Bluetooth Tracker Aero Off & 30.27 & 39.00 & 17.00 & 22.00 & 23.00 & $* *$ \\
\hline PC 1 Winforms RAT5 Gaming Mouse Aero Off & 26.56 & 36.00 & 22.00 & 17.00 & 21.00 & 21.00 \\
\hline PC 2 Unity Oculus Rift Tracker Aero Off & 27.27 & 50.00 & 14.00 & 25.00 & 26.00 & 35.00 \\
\hline PC 2 Unity Mouse Aero Off & 87.46 & 96.00 & 78.00 & 83.00 & 80.00 & 87.00 \\
\hline PC 3 OptiTrack Arena Raw Targets Aero Off & 55.00 & 60.00 & 51.00 & 40.00 & 40.00 & $*$ \\
\hline PC 3 OptiTrack Motive Raw Targets Aero Off & 52.00 & 56.00 & 46.00 & 43.00 & 43.00 & $*$ \\
\hline PC 3 OptiTrack Motive Rigid Body Aero Off & 50.43 & 54.00 & 46.00 & 48.00 & 46.00 & $*$ \\
\hline
\end{tabular}

Oculus Rift SDK were used to control the camera, and modified to support input from the mouse, so the same configuration is used for both the mouse and Rift tracker measurements. The measurements of the OptiTrack system were taken from the preview window of the included software (Arena 1.8.6 \& Motive 1.0.1) and include the rendering time of that application.

It has been asserted that the Aero feature of Windows Vista and 7, can introduce a frame of delay [13]. We found this was the case for both Winforms, and OpenGL applications, windowed and full screen, with Aero introducing $\sim 20 \mathrm{~ms}$ of latency with a refresh rate of $60 \mathrm{~Hz}$.

\section{Conclusions}

Latency is of high concern to those who engineer virtual environments. We have investigated and demonstrated the accuracy of two measurement methods, both of which are easy to use and compatible with a wide range of VEs. Both methods operate by sensing motion, resulting in signals which describe the change in position of targets over time. The phase shift that minimises the difference between these defines the latency. By cross-correlating virtual waveforms of various frequencies and phases, we have shown high invariance with low spatial (20 pixels) and temporal $(25 \mathrm{fps})$ resolutions. This suggests that the predominant source of estimation error will be spatial error in the recovered motion from the sensing devices or their pre-processing algorithms. We constructed a mechanical simulator of which the latency could be finely controlled, and used it to investigate Steed's Method using cameras, and Di Luca's Method using photodiodes. Both were found to be highly accurate. Subsampling the captures taken for Steed's Method in the spatial domain reinforced what we found with virtual data - that spatial resolution has no significant interaction with accuracy. Given this, we subsampled low resolution $(224 \times 64)$ high speed video in the temporal domain and found again the empirical measurements corroborated what we found with virtual data. In order to verify operation of the mechanical simulator the Automated Frame Counting method using high speed video was used. In theory the latency is directly observable with discrete frames and the accuracy is unquestionable when frame counting. In practice we discovered what others had found in that manual frame counting is time consuming and far from error free due to the difficulty in determining motion from noise. Consequently, we present an automated frame counting method based on image processing techniques (blob tracking and edge detection). We prove the concept with virtual data, and verify the operation of the mechanical simulator and the method in practice through demonstrating the high correlation between intended latency and estimated latency when both operate together. We present this method as a third latency measurement technique, with the advantage that it can measure changes in latency over relatively small amounts of time. If we consider the worst case 99\% CI mechanical simulator actuation $(\sim 2 \mathrm{~ms})+$ automated frame counting estimation error $(\sim 1 \mathrm{~ms})$, we could state with confidence the Automated Frame Counting method has an accuracy of $\sim 3 \mathrm{~ms}$. This is similar to the average $99 \% \mathrm{CI}$ across the various frequencies under test, for Steed's Method $(\sim 7 \mathrm{~ms})$ and Di Luca's Method ( $\sim 8 \mathrm{~ms})$. With these levels of confidence we measure a number of interactive systems. We find as expected the cross-correlation of motion from video, at $1000 \mathrm{fps}$ and $25 \mathrm{fps}$ results in deviations in the estimations of a few ms (within measurement error), and these estimations correlate strongly with the average latency estimated using Automated Frame Counting. Di Luca's Method deviates further from the latency estimated by Steed's method, but is generally within the range found by the Automated Frame Counting method, suggesting it tends towards one extreme or the other (or perhaps the latency encountered during the movement at the dominant frequency the algorithm band-passes on). Automated Frame Counting demonstrated the highest accuracy, but capturing VEs with high-speed video introduced complications not found with the low-speed video used in Steed's Method. For example, the low resolution required the tracker to be physically close to the display, and the tracked object in the scene be larger than would have normally been necessary. Additional lighting was also needed. With a suitable object tracking algorithm, both Automated Frame Counting and Steed's Method could operate without any modification to the VE, as they would be capable of tracking existing salient objects within a scene. The objects and environments to which they apply however are limited. To be suitable a target object must be free in space with a linear relationship between its acceleration and that of the tracker. If the VE alters the behaviour of the object in any way (e.g. damping its movement, or altering its trajectory due to it colliding with another object in the scene) the latency measurement would be inaccurate. Di Luca's Method's specialised hardware makes demands of the display technology and scene that camera-based methods do not. It also overcomes limitations inherent to these methods. It may be the only applicable technique when, for example, the tracker and display are physically far apart, the tracker is not visibly salient, or items in the scene cannot be reliably tracked (as we have already encountered).

In conclusion, any of the three latency measurement methods could accurately characterise the latency of a VE. We found Steed's Method to be easiest to use however, and the gains in accuracy provided by more specialised hardware to be no greater than the inherent variations found in most of the real systems above.

The manuals \& modified source code for all techniques and the mechanical simulator can be found at www.cs.ucl.ac.uk/staff/s.friston/.

\section{ACKNOWLEDGMENTS}

The authors would like to thank Dr. William Steptoe for his help measuring the latency of a number of VE systems.

\section{REFERENCES}

[1] Bernard D. Adelstein, Eric R. Johnston, and Stephen R. Ellis. Dynamic Response of Electromagnetic Spatial Displacement Track- 
ers. Presence (Cambridge, Mass.), 5(3):302-18, 1996.

[2] Bernard D. Adelstein, Thomas G. Lee, and Stephen R. Ellis. Head Tracking Latency in Virtual Environments: Psychophysics and a Model. Proceedings of the Human Factors and Ergonomics Society Annual Meeting, 47(20):2083-2087, October 2003.

[3] Paul Bao, Lei Zhang, and Xiaolin Wu. Canny Edge Detection Enhancement by Scale Multiplication. IEEE transactions on pattern analysis and machine intelligence, 27(9):1485-90, September 2005.

[4] Timothy J. Buker, Dennis A. Vincenzi, and John E. Deaton. The Effect of Apparent Latency on Simulator Sickness While Using a See-Through Helmet-Mounted Display: Reducing Apparent Latency With Predictive Compensation. Human Factors: The Journal of the Human Factors and Ergonomics Society, 54(2):235249, January 2012.

[5] Kuan-Ta Chen, Yu-Chun Chang, Po-Han Tseng, Chun-Ying Huang, and Chin-Laung Lei. Measuring the latency of cloud gaming systems. Proceedings of the 19th ACM international conference on Multimedia - MM '11, page 1269, 2011.

[6] Massimiliano Di Luca. New Method to Measure End-to-End Delay of Virtual Reality. Presence, 19(6):569-584, December 2010.

[7] Chris Gunn, Matthew Hutchins, and Matt Adcock. Combating Latency in Haptic Collaborative Virtual. Presence, 14(3):313328, 2005.

[8] Ding He, Fuhu Liu, Dave Pape, Greg Dawe, and Dan Sandin. Video-Based Measurement of System Latency. International Immersive Projection Technology Workshop, 2000.

[9] Caroline Jay, Mashhuda Glencross, and Roger Hubbold. Modeling the effects of delayed haptic and visual feedback in a collaborative virtual environment. ACM Transactions on Computer-Human Interaction, 14(2):8-es, August 2007.

[10] Jason Jerald, Tabitha Peck, Frank Steinicke, and Mary Whitton. Sensitivity to scene motion for phases of head yaws. In Proceedings of the 5th symposium on Applied perception in graphics and visualization - APGV '08, page 155, New York, New York, USA, 2008. ACM Press.

[11] Jason J Jerald. Scene-Motion- and Latency-Perception Thresholds for Head-Mounted Displays. PhD thesis, University of North Carolina at Chapel Hill, 2010.

[12] Tony Lindeberg. Edge Detection and Ridge Detection with Automatic Scale Selection. International Journal of Computer Vision, 30(2):117-154, 1998.

[13] Joe Ludwig. Lessons learned in porting Team Fortress 2 to Virtual Reality. Game Developers Conference, 2013.

[14] I. Scott MacKenzie and Colin Ware. Lag as a determinant of human performance in interactive systems. In Proceedings of the SIGCHI conference on Human factors in computing systems - CHI '93, pages 488-493, New York, New York, USA, 1993. ACM Press.

[15] Michael Meehan, Sharif Razzaque, Mary C. Whitton, and Frederick P. Brooks Jr. Effect of latency on presence in stressful virtual environments. Proceedings of the IEEE Virtual Reality 2003, 2003:141, 2003.

[16] Dorian Miller and Gary Bishop. Latency meter: a device endto-end latency of VE systems. Electronic Imaging 2002, pages 458-464, 2002.
[17] Joel D. Miller, Mark R. Anderson, Elizabeth M. Wenzel, and Bryan U. McClain. Latency measurement of a real-time virtual acoustic environment rendering system. Proceedings of the 2003 International Conference on Auditory Display, Boston, MA, 6-9 July 2003, 2003.

[18] Mark R. Mine. Characterization of end-to-end delays in headmounted display systems. Technical report, University of North Carolina at Chapel Hill, 1993.

[19] Mark S. Nixon and Alberto S. Aguado. Feature Extraction and Image Processing. Newnes, 2002.

[20] Nobuyuki Otsu. A Threshold Selection Method from Gray-Level Histograms. IEEE Transactions on Systems, Man, and Cybernetics, 9(1):62-66, 1979.

[21] Giorgos Papadakis, Katerina Mania, and Eftichios Koutroulis. A system to measure, control and minimize end-to-end head tracking latency in immersive simulations. VRCAI' 11 Proceedings of the 10th International Conference on Virtual Reality Continuum and Its Applications in Industry, pages 581-584, 2011.

[22] David Roberts, Toby Duckworth, Carl Moore, Robin Wolff, and John O'Hare. Comparing the End to End Latency of an Immersive Collaborative Environment and a Video Conference. 2009 13th IEEE/ACM International Symposium on Distributed Simulation and Real Time Applications, pages 89-94, 2009.

[23] Mehmet Sezgin and Bulent Sankur. Survey over image thresholding techniques and quantitative performance evaluation. Journal of Electronic Imaging, 13(1):220, January 2004.

[24] Tobias Sielhorst, Wu Sa, Ali Khamene, Frank Sauer, and Nassir Navab. Measurement of absolute latency for video see through augmented reality. 2007 6th IEEE and ACM International Symposium on Mixed and Augmented Reality, pages 1-4, November 2007.

[25] Mel Slater, Beau Lotto, Maria Marta Arnold, and Maria V Sanchez-Vives. How we experience immersive virtual environments: the concept of presence and its measurement. Anuario de Psicología, 40(2):193-210, 2009.

[26] Anthony Steed. A Simple Method for Estimating the Latency of Interactive, Real-Time Graphics Simulations. Proceedings of the 2008 ACM symposium on Virtual Reality Software and Technology, pages 123-129, 2008.

[27] Colin Swindells, John C Dill, and Kellogg S Booth. System lag tests for augmented and virtual environments. In Proceedings of the 13th annual ACM symposium on User interface software and technology - UIST '00, pages 161-170, New York, New York, USA, 2000. ACM Press.

[28] Tinne Tuytelaars and Krystian Mikolajczyk. Local Invariant Feature Detectors: A Survey. Foundations and Trends in Computer Graphics and Vision, 3(3):177-280, 2007.

[29] O. R. Vincent and O. Folorunso. A descriptive algorithm for sobel image edge detection. Proceedings of Informing Science \& IT Education Conference (InSITE), 2009.

[30] Weixin Wu, Yujie Dong, and Adam Hoover. Measuring Digital System Latency from Sensing to Actuation at Continuous 1-ms Resolution. Presence: Teleoperators and Virtual Environments, 22(1):20-34, 2013.

[31] Paul Zimmons and Abigail Panter. The Influence of Rendering Quality on Presence And Task Performance in a Virtual Environment. Proceedings of the IEEE Virtual Reality 2003, pages 293-294, 2003. 